\title{
Les Lettres d'un voyageur de George Sand
}

Une poétique romantique du paysage

Yvon Le Scanff

\section{OpenEdition}

\section{Journals}

Édition électronique

URL : http://journals.openedition.org/recherchestravaux/230

DOI : 10.4000/recherchestravaux.230

ISSN : 1969-6434

Éditeur

UGA Éditions/Université Grenoble Alpes

Édition imprimée

Date de publication : 15 avril 2007

Pagination : 167-180

ISBN : 978-2-84310-107-7

ISSN : 0151-1874

\section{Référence électronique}

Yvon Le Scanff, « Les Lettres d'un voyageur de George Sand », Recherches \& Travaux [En ligne], 70 | 2007, mis en ligne le 04 mars 2009, consulté le 10 décembre 2020. URL : http://

journals.openedition.org/recherchestravaux/230 ; DOI : https://doi.org/10.4000/recherchestravaux. 230 


\section{Les Lettres d'un voyageur de George Sand Une poétique romantique du paysage}

En tant que récits de voyage, ou plutôt "impressions " (préface ; p. 646 ; p. $38^{1}$ ) de voyage, cette " œuvre informe" (préface ; p. 649 ; p. 41) pose la question des conditions de possibilité et de réalisation de la description dans le cadre d'une réflexion sur l'art du paysage littéraire. La poétique sandienne est d'autant plus originale qu'elle se fonde sur une tension créatrice à l'endroit de la description de paysage. Elle met en ouvre une tension entre l'objet naturel et le sujet, labile, et sans doute expérimental ${ }^{2}$, qui se présente comme auteur et donc responsable de la description. Cette tension romantique entre objectivisme et subjectivisme, est redoublée, chez Sand, par une réflexion sur les ambitions littéraires de la description : les esthétiques du pittoresque et du sublime sont alors sollicitées pour proposer une poétique originale du paysage littéraire. Ainsi les solutions esquissées sur le plan de l'écriture fondent l'originalité de la démarche sandienne et l'inscrivent au cœur de la problématique esthétique du romantisme.

\section{La description pittoresque : l'esthétique du tableau}

Les ressources de l'art pittoresque consistent d'abord à proposer à l'écrivain voyageur, sur le modèle bien connu de l'ut pictura poésis horacien, un modèle indépassable et inégalable, celui de la peinture : il s'agit de s'inspirer de l'art

1. Selon la convention que nous adoptons pour ce volume: G. Sand, Lettres d'un voyageur, pages de l'édition de G. Lubin puis pages de l'édition de $\mathrm{H}$. Bonnet.

2. Voir P. Laforgue, Corambé. Identité et fiction de soi chez George Sand, op. cit., notamment les p. 75-95 consacrées aux Lettres d'un voyageur. 
pictural et de composer davantage des tableaux que de simples descriptions ${ }^{3}$. Le lexique rend compte de cette tentative de picturalisation de la description : Sand utilise fréquemment le terme de " tableau ${ }^{4}$ " pour désigner ses paysages littéraires. Le pittoresque permet évidemment de délimiter la description à la façon d'une œuvre picturale et de lui donner un cadre, ce sera par exemple celui d'une fenêtré à Bassano :

Je m'éveillai au lever du soleil, et je vis de ma fenêtre, s'élever, dans le bleu vif de l'air, les créneaux enveloppés de lierre de l'antique forteresse qui domine la vallée. (Lettre I ; p. $651 ;$ p. $42^{6}$.)

Le point de vue peut également se présenter comme panoramique et le paysage est alors défini comme l'ensemble d'un pays qu'embrasse le regard du Voyageur :

Au coucher du soleil, je me trouvai au faîte d'une crête de rochers ; c'était la dernière des Alpes. À mes pieds s'étendait la Vénétie, immense, éblouissante de lumière et d'étendue. J'étais sorti de la montagne, mais vers quel point de ma direction ? Entre la plaine et le pic d'où je la contemplais s'étendait un beau vallon ovale, appuyé d'un côté au flanc des Alpes, de l'autre élevé en terrasse au-dessus de la plaine et protégé contre les vents de la mer par un rempart de collines fertiles. Directement au-dessous de moi, un village était semé en pente dans un désordre pittoresque. (Lettre II ; p. 674 ; p. 64.)

L'élection d'un point de vue et d'un cadre permet de construire la description à la façon d'un tableau ${ }^{7}$ selon divers plans hiérarchisés et organisés au gré d'une perspective horizontale allant du proche au lointain. Par exemple :

Un demi-cercle de collines fertiles [...] faisait un premier cadre au tableau; et les monts neigeux, étincelants aux premiers rayons du soleil, formaient, au-delà, une seconde bordure immense, qui se détachait comme une découpure d'argent sur le bleu solide de l'air. (Lettre I ; p. 653 ; p. 44.)

C'est le pittoresque qui permet d'embrasser la totalité du paysage en organisant de façon progressive l'ensemble de ses éléments selon un point de vue

3. Voir à ce sujet l'article de A. Guyot, "Peindre ou décrire : un dilemme de l'écrivain voyageur au XIX" siècle ", Recherches \& Travaux, 1997, nº52, p. 99-119.

4. Voir par exemple la lettre I ; (p. 653, 656 et 667 ; p. 44, 47 et 56) et la lettre X (p. 902 ; p. 279).

5. Anne Cauquelin parle de ce processus de "cadrage " comme d'une opération fondamentale pour l'avènement d'un paysage : voir "Le paysage par la fenêtre ", L'Invention $d u$ paysage (1989), Paris, PUF « Quadrige », 2000, p. 121-126.

6. Voir aussi la lettre X (p. 902 ; p. 279).

7. Voir sur ce point l'article de Ph. Berthier, "Lettres d'un voyageur : le cru et le cuit", op. cit., p. 141 : «Il arrive qu'un paysage, dans l'aménagement de ses plans et la disposition de ses accidents, réponde tellement, par le jeu d'un hasard talentueux, aux canons du pittoresque qu'il fasse tableau ". 
cohérent, selon une perspective pyramidale et verticale qui convient parfaitement à la représentation de la montagne :

Toute la partie inférieure du vallon est soigneusement cultivée. Plus haut s'étendent d'immenses pâturages dont la nature prend soin elle-même. Puis une rampe de rochers arides s'élève jusqu'aux nuages, et la neige s'étale au faîte comme un manteau. (Lettre I ; p. 656 ; p. 46.)

Les théoriciens et voyageurs définissent également le pittoresque comme une catégorie esthétique propre à rendre les effets de la nature, c'est-à-dire l'irrégularité, voire la rudesse des formes, le caractère accidenté du paysage, la variété des aspects et des reliefs. Le " désordre pittoresque » dont parlait Sand est l'expression de la diversité naturelle dans l'unité du tableau descriptif. Le regard pittoresque prend possession de la variété, de la complexité, voire de l'irrégularité naturelle et les convertit en plaisir esthétique :

[...] j'embrassai ce riche aspect de la Vénétie pendant plusieurs lieues, sans être fatigué de son immensité, grâce à la variété des premiers plans, qui descendent par gradins de monticules et de ravines jusqu'à la surface unie de la plaine. (Lettre I ; p. 677 ; p. 66.)

Cette approche du paysage maintient l'intérêt et la curiosité du Voyageur en lui ménageant un certain nombre d'accidents qui se transforment en péripéties dramatiques, voire en "scènes théâtrales " dont parle ClaudeHenri Watelet ${ }^{8}$. À la fin de la lettre VIII (p. 868-869 ; p. 247), qui évoque Valençay, George Sand fait l'éloge de ce genre de paysage : "Ce lieu est un des plus beaux de la terre, et aucun roi ne possède un parc plus pittoresque [...]. » Ce " vallon frais et touffu " ménage des surprises : c'est une " oasis » perdue « au milieu des tristes plaines qui l'environnent et qui n'en laissent pas soupçonner l'approche ». Le terrain est scandé par des coups de théâtre paysagers :

On tombe tout à coup dans un ravin hérissé de rochers et de forêts, dans des jardins royaux du milieu desquels s'élève un palais espagnol élégant et poétique, qui se mire du haut des rochers dans les eaux d'une rivière bleue. Il semble qu'on soit arrivé en rêve dans quelque pays enchanté, qui doit s'évanouir au réveil et qui s'évanouit en effet au bout d'un quart d'heure lorsqu'on traverse seulement le vallon et qu'on suit la route du midi. Les plaines sans fin, les bruyères jaunes, les horizons plats et nus reparaissent. (P. 868 ; p. 247.)

Les plans du tableau se transforment en scènes dramatiques dès que le point de vue devient dynamique, dès que la description devient promenade.

8. Cl.-H. Watelet, Essai sur les jardins (1774), Saint-Pierre-de-Salerne, Gérard Monfort, 2004, p. 23-24. 
Le paysage pittoresque, en alliant les exigences de la construction picturale et les tentations du dynamisme théâtral, peut alors se concevoir comme décor : "Nous arrivâmes à ces carrières abandonnées qui s'encadrent dans la plus riche verdure, et dont les profondeurs offrent une décoration vraiment théâtrale» (p. 869 ; p. 247).

Cette appréhension du paysage par la catégorie esthétique du pittoresque est également présente dans les Lettres d'un voyageur, en tant que transpositions d'art, " artialisation ${ }^{9}$ " et " picturalisation du pays ${ }^{10}$ " pour reprendre les formules d'Alain Roger. Il peut signifier qu'il faut voir la nature comme un peintre, comme un artiste; et le pittoresque consiste alors à relever ce qui est digne de la peinture dans un paysage naturel : « Le mot pittoresque s'applique à tout objet, et à toute sorte de paysage qui a été représenté avec avantage en peinture, ou pourrait l'être ${ }^{11}$ ». Mais le pittoresque se définit aussi de façon plus extensive comme un mode de vision de la nature qui privilégie l'art, les associations entre la réalité et ses représentations artistiques :

[...] ce rapport même avec la peinture, exprimé par le mot pittoresque, est celui qui apporte tout le plaisir tiré des associations d'idées ; plaisir qui peut donc être ressenti uniquement par les personnes possédant une certaine connaissance de cet art. ${ }^{12}$

L'épisode de Possagno, dans la première lettre, montre comment un paysage s'associe, comme le dit Sand, à une "idée de personnification ", à une association d'idées qui en conditionne la représentation. Le paysage ne prend sens qu'en fonction de la référence à Canova : l'église, « beau et vaste temple de marbre tout neuf, éclatant de blancheur " est bien sûr "l'œuvre de

9. Voir A. Roger, "Ut pictura hortus. Introduction à l'art des jardins", Mort du paysage?, F. Dagognet (dir.), Seyssel, Champ Vallon, 1982, p. 96-97 : « Notre perception esthétique de la nature est toujours médiatisée par une opération artistique. [...] Un site naturel n'est esthétiquement perçu qu'à partir et à travers un paysage, qui exerce donc, en ce domaine, la fonction d'artialisation. "Voir aussi du même auteur, Court traité du paysage, Paris, Gallimard "Bibliothèque des sciences humaines ", 1997, chap. I, p. 11-30.

10. A. Roger, "Esthétique du paysage au siècle des Lumières ", Composer le paysage. Constructions et crises de l'espace (1789-1992), Seyssel, Champ Vallon, 1989, p. 66.

11. U. Price, Essais sur le Pittoresque, comparé au Sublime et au Beau; et sur l'intérêt d'étudier les tableaux, afin d'embellir les paysages naturels (1794), cité dans Art et nature en GrandeBretagne au XVIIT siècle, textes présentés par M.-M. Martinet, Paris, Aubier, 1980, p. 249. Claude-Henri Watelet disait la même chose dès 1774 : le pittoresque, "comme le désigne le nom tient aux idées de la peinture " (Essai sur les jardins, op. cit., p. 23).

12. R. Payne Knight, Recherches analytiques sur les principes du Goût (1808), cité dans Art et nature en Grande-Bretagne au XVIII siècle, op. cit., p. 271. 
Canova "(p. 675 ; p. 64). Plus profondément, le génie du lieu témoigne de l'inspiration de l'artiste :

Les habitants de Possagno disent avec orgueil que les principaux modèles de la riche collection des œuvres de Canova sont sortis de leur vallée. Il suffit en effet de la traverser pour y retrouver, à chaque pas, le type de froide beauté qui caractérise la statuaire de l'Empire. (P. 676 ; p. 65.)

Le paysage tout entier devient alors un paysage inspiré : "Le vallon de Possagno a la forme d'un berceau : il est fait à la taille de l'homme qui en est sorti. [...] La vie de Canova fut féconde et généreuse comme le sol de sa patrie » (p. 676 ; p. 64-65). Il est alors paysage idéal et se présente comme un idéal selon la poétique sandienne, c'est-à-dire clos et protecteur mais ouvert sur l'infini :

Cette espèce de paradis terrestre, où la jeunesse intellectuelle peut s'épanouir avec toute sa sève printanière, cet horizon immense qui semble appeler les pas et les pensées de l'avenir, ne sont-ce pas là deux conditions principales pour le déploiement d'une belle destinée ? (P. 675-676 ; p. 65.)

Cependant, la poétique sandienne ne peut être réduite à une simple sensibilité pittoresque à l'égard du paysage. Par son ambition toute romantique et par l'intérêt qu'elle porte au paysage, elle excède la représentation d'un simple objet et se présente comme expérience intégrale du sujet.

\section{L'idéal romantique : subversion et transgression des limites du pittoresque}

L'art pittoresque est un art du visible, de l'objectivité sensible qui cherche essentiellement à plaire aux yeux, à enchanter le regard et pour cela ne s'en tient qu'à l'apparence extérieure : il ne parle pas à l'âme, il ne touche pas l'imagination. Le pittoresque souffre selon Sand de cette stricte orientation artistique et picturale et le projet sandien ne se satisfait pas d'une telle esthétique. Le souci du pittoresque n'est donc pas suffisant pour qui cherche à éprouver dans l'appréhension du paysage, dans l'émotion esthétique, une communion et une harmonie avec l'ensemble de ses facultés : "les créations de l'art parlent à l'esprit seul, et [...] le spectacle de la nature parle à toutes les facultés » (lettre I ; p. 652 ; p. 43). George Sand reformule et reprend une distinction d'importance que René-Louis de Girardin avait déjà établie, en 1777, dans De la composition des paysages : seule la nature a le pouvoir d'offrir

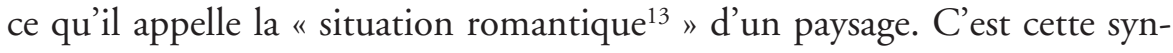

13. R.-L. de Girardin, De la composition des paysages (1777), Seyssel, Champ Vallon, 1992, p. 99. 
thèse romantique des facultés, c'est cette résolution du sensible, l'union du sentiment et de la sensation, de l'apparence et de l'idéalité que George Sand semble rechercher dans son approche littéraire du paysage. La lettre IX est claire sur ce point : le poète doit être "artiste » et avoir le "sens du pittoresque ", mais il doit être également " philosophe " et "sonder les profondeurs du monde idéal » :

Quand ce développement de la faculté de voir, de comprendre et d'admirer ne s'applique qu'aux objets extérieurs, on n'est qu'un artiste; quand l'intelligence va au-delà du sens pittoresque, quand l'âme a des yeux comme le corps, quand elle sonde les profondeurs du monde idéal, la réunion de ces deux facultés fait le poète ; pour être vraiment poète, il faut donc être à la fois artiste et philosophe. (P. 872 ; p. 251.)

Girardin définissait déjà le caractère romantique, qui intéresse " tout à la fois l'œil et l'esprit ${ }^{14}$ ", comme synthèse du pittoresque et du poétique, comme "situation » et "impression " d'un paysage ${ }^{15}$. Il s'agit donc de voir, de composer des vues plus ou moins objectives, mais aussi et surtout de concevoir des visions subjectives, qui sont autant d'expressions proprement romantiques de la nature, c'est-à-dire d'impressions du contemplateur pensif. Cet au-delà du pittoresque que George Sand revendique dans sa poétique est le supplément d'âme romantique par excellence : "le pittoresque ne s'applique encore qu'à l'aspect objectif d'une représentation de la nature ", analysera Hans Robert Jauss, mais ce qui " donne un caractère romantique, c'est bien moins la beauté objective de la nature que l'impression subjective $^{16}$ " qu'elle produit. C'est bien dans cette tension que réside le problème fondamental de la poétique sandienne du paysage dans les Lettres d'un voyageur. En effet, la quête de l'idéal, de l'idée du paysage, présente le risque d'une perte des apparences au profit d'un subjectivisme absolu, que Sand revendique par ailleurs comme principe de l'écriture de ses lettres de voyage :

Ne lis jamais mes lettres avec l'intention d'y apprendre la moindre chose sur les objets extérieurs ; je vois tout au travers des impressions personnelles. Un voyage n'est pour moi qu'un cours de psychologie et de physiologie dont je suis le sujet, soumis à toutes les épreuves et à toutes les expériences qui me tentent [...]. (Lettre X; p. 893 ; p. 271.)

Cette tension entre l'idéal et le réel, entre l'invisible et le visible, entre la représentation et l'impression a pour conséquence une certaine surestimation

14. Ibid., p. 21.

15. Ibid., p. 99.

16. H. R. Jauss, "La modernité dans la tradition littéraire », Pour une esthétique de la réception (1978), Paris, Gallimard «Tel », 1990, p. 191. 
de la faculté imaginative dans ce qu'elle a de fantaisiste ${ }^{17}$. La description sandienne devient un facteur de destruction des formes et en même temps le vecteur de l'imagination créatrice, de la "vision transformante ${ }^{18}$ » :

Quand les formes s'effacent, quand les objets semblent trembler dans la brume, quand mon imagination peut s'élancer dans un champ immense de conjectures et de caprices, quand je peux, en clignant un peu de la paupière, renverser et bouleverser une cité, en faire une forêt, un camp ou un cimetière ; [...] alors je jouis vraiment de la nature, $j$ 'en dispose à mon gré, je règne sur elle, je la traverse d'un regard, je la peuple de mes fantaisies. (Lettre II ; p. 684 ; p. 73.)

Elle aboutit au fait qu'il faille paradoxalement fermer les yeux pour composer un paysage, comme le Voyageur l'admet à la fin de ses lettres vénitiennes : "Je fermai les yeux, comme je fais souvent, pour résumer les diverses impressions de ma promenade, et me composer une vue générale du paysage que je venais de parcourir » (lettre III ; p. 734 ; p. 121). Mais alors, le paysage en question, celui de Torcello, disparait complètement dans cette épreuve. De la même façon, à Oliero, le Voyageur profite de l'absence de particularités pittoresques : "Je traversai ce désert sans rencontrer un seul accident qui dérangeât mon rêve $[\ldots]$ » (lettre I ; p. 673 ; p. 63). Le paysage naturel suscite alors une inspiration proprement onirique et fantaisiste dans laquelle il s'abolit néanmoins entièrement en tant que représentation sensible :

Je fermai les yeux au pied d'une roche, et mon esprit se mit à divaguer. En un quart d'heure, je fis le tour du monde ; et quand je sortis de ce demi-sommeil fébrile, je m'imaginais que j'étais en Amérique, dans une de ces éternelles solitudes que l'homme n'a pu conquérir encore sur la nature sauvage. (Lettre I ; p. $673 ;$ p. 62.)

Le paysage devient illusion, hallucination ou vision : il n'est plus soumis à un regard, ou à un point de vue qui en compose les limites sensibles. La poétique sandienne glisse donc du pittoresque au romantique, « du déterminé à l'indéterminé19 ». Le paysage devient d'autant plus intéressant pour l'âme qu'il disparaît pour les yeux : Venise sombre dans l'obscurité et le rêve et devient alors un objet digne d'être appréhendé par le poète qui en transfigure l'apparence par la fantaisie et par le fantastique :

17. À propos de la fantaisie romantique, nous renvoyons aux analyses de Pierre Laforgue dans Romanticoco. Fantaisie, chimère et mélancolie (1830-1860), op. cit., notamment p. 109118 (sur les Lettres d'un voyageur). P. Laforgue note justement que « la fantaisie n'est ni gratuite ni arbitraire, elle est la modalité rêveuse de la réalité »(p. 115).

18. Voir M. Raymond, "George Sand, la rêverie et la vision transformante ", Romantisme et rêverie, Paris, José Corti, 1978, p. 187-205.

19. Voir G. Poulet, "George Sand ", La Pensée indéterminée, Paris, PUF "Écriture ", 1987, t. II, p. 77. 
Jamais je n'avais vu Venise si belle et si féerique. Cette noire silhouette, jetée entre le ciel et l'eau ardente comme dans une mer de feu, était alors une de ces sublimes aberrations d'architectures que le poète de l'Apocalypse a dû voir flotter sur les grèves de Patmos quand il rêvait sa Jérusalem nouvelle [...].

Peu à peu les couleurs s'obscurcirent, les contours devinrent plus massifs, les profondeurs plus mystérieuses. Venise prit l'aspect d'une flotte immense, puis d'un bois de hauts cyprès où les canaux s'enfonçaient comme de grands chemins de sable argenté. (Lettre II ; p. 684 ; p. 72-73.)

De la même façon, la description du massif du Mont-Blanc, grâce à la " nuit ", à la "vapeur d'argent " et à la "fumée noire " de feux agricoles, devient un "spectacle magnifique ». Le réalisme de la notation cède volontiers à la tentation du fantastique : le paysage se perd et se confond, la "vallée » est " plongée et perdue » dans l'indistinct et l'informe :

Au-dessus des feux, au-dessus de la fumée et de la brume, la chaîne du MontBlanc montrait une de ces dernières ceintures granitiques, noire comme l'encre et couronnée de neige. Ces plans fantastiques du tableau semblaient nager dans le vide. (Lettre X; p. 902 ; p. 279.)

Cet idéalisme s'inscrit selon Sand dans le cadre de l'émergence de la modernité romantique géographiquement et mythiquement située au nord, en Allemagne, qui s'oppose au naturalisme italien et à sa prédominance visuelle. Le débat entre le Voyageur et son correspondant qu'engage le début de la lettre II met en scène cette opposition entre d'une part, les " grandes lignes pures ", les contours hardiment dessinés " la " lumière riche et splendide ", les « couleurs » et les « formes » des arts de la « vieille Italie »; et d'autre part, les « idées fantastiques » allemandes qui estompent la représentation du réel à la façon d'une "éponge trempée dans les brouillards du Nord ". Au "vrai beau » classique et à sa contemplation sereine s'opposent donc la rêverie romantique et sa passion du vague. Dans ce glissement progressif du plaisir descriptif, le paysage se présente comme signe dans (et de) l'écriture à mesure qu'il s'absente en tant que référent et référence du Voyageur. Sa disparition effective est certes une sorte d'assomption littéraire, mais la description sandienne se présente néanmoins comme une "opération essentiellement négative ${ }^{20} »$.

Dans les Lettres d'un voyageur, cette négativité est assumée par une stratégie romantique de l'effet : par une fantaisie subversive et transgressive, mais aussi par une poétique qui va recourir à l'esthétique du sublime afin de pallier

20. Loc. cit. 
l'absence de positivité de la description. Les Lettres d'un voyageur montrent une telle mise en ouvre littéraire : le privilège des "objets négatifs" (Chateaubriand) $^{21}$, de la privation sensible (Burke) ${ }^{22}$ impose bien le sublime comme sentiment, effet, impression. Elle implique le primat de la rêverie, c'est-à-dire du sentiment et non seulement de la simple sensation pittoresque. La description de paysage devient vision sublime :

La lune s'élevait peu à peu et commençait à montrer sa face curieuse au-dessus des toits ; elle aussi avait l'air d'écouter et d'aimer cette musique. Une des rives de palais du canal, plongée encore dans l'obscurité, découpait dans le ciel ses grandes dentelles mauresques, plus sombres que les portes de l'enfer. L'autre rive recevait le reflet de la pleine lune, large et blanche alors comme un bouclier d'argent, sur ses façades muettes et sereines. Cette file immense de constructions féeriques, que n'éclairait pas d'autre lumière que celle des astres, avait un aspect de solitude, de repos et d'immobilité vraiment sublime ${ }^{23}$. (Lettre II ; p. 707 ; p. 94.)

Le sublime se présente comme une gageure pour la description de paysage : sa négativité empêche toute emprise de la sensation pittoresque, toute appréhension purement sensible. Il met en déroute la sensibilité et met en échec les pouvoirs de l'imagination. Le seul mode d'appréhension semble encore en être la rêverie. La description de paysage glisse vers la méditation. La négativité sublime libère l'âme de toute distraction pittoresque, l'œil se perd dans le vide, l'infini, l'indéfini et l'écriture devient le miroir pensif d'un paysage extasié :

La contemplation des cimes immobiles du mont Blanc, l'aspect de cette neige éternelle, immaculée, sublime de blancheur et de calme, avaient suffi pendant trois ou quatre jours du mois dernier, pour donner à mon âme une sérénité inconnue depuis longtemps. (Lettre IV ; p. 739-740 ; p. 126 24 .)

21. Voir F.-R. de Chateaubriand, Génie du christianisme, Paris, Gallimard "Bibliothèque de la Pléiade ", 1978, livre II, chap. X, p. 675 : "Les images favorites des poètes enclins à la rêverie sont presque toujours empruntées d'objets négatifs, tels que le silence des nuits, l'ombre des bois, la solitude des montagnes, la paix des tombeaux, qui ne sont que l'absence de bruit, de la lumière, des hommes et des inquiétudes de la vie."

22. Voir E. Burke, Recherche philosophique sur l'origine de nos idées du Sublime et du Beau, trad. B. Saint-Girons, Paris, Vrin, 1990, livre II, chap. VI, p. 115: "Toutes les privations générales sont grandes parce qu'elles sont toutes terribles : la vacuité, l'obscurité, la solitude et le silence ".

23. Comment ne pas rapprocher cette magnifique évocation de ce que Goethe disait du paysage romantique : "Ce que l'on appelle le caractère romantique d'un paysage, c'est le calme sentiment du sublime sous la forme du passé ou, ce qui revient au même, de la solitude, de l'absence, de l'isolement ", cité dans H. R. Jauss, Pour une esthétique de la réception, op. cit., p. 193.

24. Voir également la fin de la lettre VII et l'évocation des " neiges sublimes du mont Blanc» (p. 850 ; p. 230), virtualités idéales d'une vie vouée à l'absolu extatique de la beauté et de l'art. 
Cette prédilection pour la négativité permet même la surestimation ou la valorisation des paysages caractérisés par la sévérité et l'austérité. Dans le Tyrol, les «solitudes d'une incroyable mélancolie ", les « lieux incultes, inhabitables, qui n'appartiennent à personne, que l'on aborde difficilement, et d'où il semble impossible de sortir » voient leur négativité se renverser en généralité sublime, antipittoresque :

Je m'arrêtai dans un certain amphithéâtre de rochers auquel pas une construction, pas un animal, pas une plante ne donnait de physionomie particulière. Il en avait une terrible, austère, désolée, qui n'appartenait à aucun pays, et qui pouvait ressembler à toute autre partie du monde qu'à l'Italie. (Lettre I; p. 673; p. 62.)

On entre alors dans une autre catégorie d'appréhension esthétique du paysage, celle du sublime : «La nature n'est pas belle ainsi, mais elle est solennelle [...] " (lettre VI ; p. 800 ; p. 182). Le paysage devient abstrait, impossible, indescriptible et ne peut s'appréhender que par l'absence de tout contenu naturel :

Il fait une nuit singulièrement mélancolique ; un ciel gris, des étoiles faibles et voilées, pas un souffle dans les plantes, une impénétrable obscurité sur la terre. Les grands sapins élèvent leurs masses noires et vagues dans l'air grisâtre. [...] Tout est silence, mystère, ténèbres ; pas une grenouille verte dans les fossés, pas un insecte dans l'herbe, pas un chien qui aboie à l'horizon, le murmure de la rivière ne nous arrive même pas ; le vent souffle du sud et l'emporte en traversant la vallée. (Loc. cit.)

Si ce paysage ne parle qu'à " un seul de nos sens », "celui dont le rossignol parle si éloquemment à un être créé pour lui », c'est au sentiment et non à la sensation qu'il s'adresse en tant qu'expression naïve du désir, de son manque et de sa plénitude. Mais " cette méthode n'est ni d'un artiste, ni d'un poète " (lettre II ; p. 685 ; p. 74) doit avouer Sand : le paysage idéal se présente comme un paysage impossible, un paysage en creux, en négatif, en fuite, vidé de toute positivité. Le risque de la disparition sensible se pose alors avec le sublime comme avec la fantaisie : le subjectivisme et l'arbitraire d'une part ; l'informe et l'abstraction de l'autre, deviennent un risque pour le "poète " qui, certes, se voulait le "philosophe " du paysage, mais qui ambitionnait aussi d'en être l'" artiste ", le garant pour les formes, grâce à l'acuité de son " sens du pittoresque ».

\section{Le paysage sandien : une synthèse romantique de l'expérience descriptive}

La première solution de ce paradoxe créateur consiste à encadrer le sublime et donc à déborder le pittoresque. Encadrer le sublime permet d'en supporter 
l'impression terrible, mais aussi l'uniformité négative ou indéfinie qui se présente comme une gageure pour la description, et une épreuve pour la sensibilité (lettre I ; p. 677 ; p. 66). Déborder le pittoresque, c'est dépasser la stricte notation référentielle, légitimer l'écriture descriptive, lui donner une autre dimension, celle de la rêverie sublime qui relie, dans un même mouvement de pensée, l'écrivain et son lecteur. Les " tableaux » sandiens analysés précédemment doivent être relus dans ce sens : ils s'ouvrent vers un infini qui en illimite les perspectives (lettre I; p. 653 et p. 656 ; p. 44 et p. 46). L'évocation de la "troisième grotte » d'Oliero, «le plus beau jardin naturel de la terre ", est exemplaire à ce sujet. Le terme de " jardin naturel ", si important pour comprendre ce que Sand entend par paysage, ou jardin idéal ${ }^{25}$, est l'expression quasi dialectique d'une synthèse entre les ambitions infinies de l'esthétique et les limites fixées par un certain eudémonisme éthique. Dans la composition de ses paysages, Sand revendique une liaison, une convergence, voire une conciliation entre l'éthique et l'esthétique, entre le vivre et le sentir, entre le bonheur et l'absolu. La description de la grotte d'Oliero se présente à la façon d'un tableau selon les règles de l'art pittoresque, le paysage lui-même est d'une composition particulièrement pittoresque ; pourtant et le tableau, et le paysage se trouvent débordés, dépassés par une perspective sublime qui, tout en faisant partie du tableau, subvertit néanmoins les limites de la représentation :

En face de la grotte, au bout d'une perspective de fleurs et de pâle verdure, jetées comme un immense bouquet que la main des fées aurait délié et secoué sur le flanc des montagnes, s'élève un géant sublime, un rocher perpendiculaire, taillé par les siècles sur la forme d'une citadelle flanquée de ses tours et de ses bastions. Ce château magique, qui se perd dans les nuages, couronne le tableau frais et gracieux du premier plan, d'une sauvage majesté. Contempler ce pic terrible, du fond de la grotte, au bord de la source, les pieds sur un tapis de violettes, entre la fraîcheur souterraine du rocher et l'air chaud du vallon, c'est un bien-être, c'est une joie que j'aurais voulu me retirer pour te l'envoyer. (Lettre I; p. 666-667; p. 56.)

La ligne de fuite du tableau qui relie le premier plan pittoresque à un arrière-plan sublime fait glisser la sensation vers le sentiment du paysage, la « faculté de voir " vers celle de rêver, de sonder les " profondeurs du monde idéal $^{26}$ ». Mais le "sens pittoresque » de l'artiste reste une nécessité pour le

25. Voir sur ce point mon article, «La poétique du jardin naturel dans Nouvelles Lettres d'un voyageur ", Fleurs et jardins dans l'ouvre de George Sand, S. Bernard-Griffiths et M.-C. Levet (dir.), Clermont-Ferrand, Presses universitaires Blaise-Pascal " Révolutions et Romantismes ", 2006, p. 103-113.

26. Au sujet de cette problématique, voir $S$. Vierne, «La montagne romantique : le réel et l'imaginaire. L'exemple de George Sand ", Eidôlon, n 54, mai 2000, Paysages romantiques, 
poète du paysage, notamment dans le cadre d'une communication épistolaire qui se veut communion avec le destinataire. Le glissement n'est donc pas abolition, le dépassement est plutôt conservation par l'enlèvement du plaisir vers la joie, ravissement de la sensation pittoresque vers le sentiment sublime. De la même façon, la contemplation distanciée et médiatisée du " pic terrible " de l'arrière-plan devient source de plaisir esthétique par la présence du « tableau frais et gracieux du premier plan ».

C’est bien là que réside la seconde solution que propose la poétique du paysage dans les Lettres d'un voyageur : élever le "terrible " au sublime par le moyen des ressources du "sens pittoresque ", c'est-à-dire rendre une certaine positivité à la négativité et accéder au sublime par-delà le terrible. Il faudrait par exemple relire la description-promenade faite dans la région d'Oliero (lettre I ; p. 673-674; p. 62-63) et l'évocation de son " désert [...] sans accident » et remarquer combien l'apparition fortuite et dramatique d'un détail pittoresque (" au détour de la montagne [...] une petite niche creusée dans le roc») transfigure ce "stérile » et terrible paysage et lui donne son sens et tout son intérêt. De la même façon, l'expérience sensible faite à la Chartreuse de Mériat $^{27}$, près de Nantua, est tout à fait exemplaire. Le site se présente d'abord comme un paysage d'horreur sublime assez conventionnel, proche du stéréotype :

La pluie fouette les sapins échevelés; des nuages courent sur les flancs de la gorge ; le brouillard enveloppe les cimes; et par mille angles du sentier qui serpente au sein des noires forêts, nous pénétrons dans une région vraiment sublime de tristesse.

G. Peylet (dir.), p. 255-268. La thèse de l'article est la suivante : Sand a besoin « au départ d'un paysage réellement vu ", mais il doit ouvrir une " porte privilégiée vers la rêverie et le rêve ", vers un " au-delà » du paysage, vers un idéal, " comme si le rêve absolu, parce qu'il ne se rattache pas à une expérience vécue, ne pouvait s'inscrire dans l'écriture sans se dévaloriser ». Les analyses de Pierre Laforgue montrent également chez Sand un jeu, au sens plein du terme, entre la réalité et sa représentation : "Cette autre réalité, qui est une réalité autre, existe non pas à l'état d'idéal, mais à l'état de réel, ou, pour employer deux concepts éminemment romantiques, à l'état d'absolu et de sublime " (Romanticoco. Fantaisie, chimère et mélancolie, op. cit., p. 115).

27. Voir l'analyse qu'en propose Philippe Berthier : "De même, les rêveries héritées du roman gothique permettent d'habiter un site par lui-même rebutant, comme celui de la Chartreuse de Mériat près de Nantua : appelée à la rescousse, la mémoire culturelle mobilise un certain répertoire spécialisé, pour intégrer au patrimoine quelque chose qui, par sa décourageante austérité, semblait devoir être inutilisable, ininterprétable. " "Lettres d'un voyageur: le cru et le cuit », op. cit., p. 141. 
Pas une figure humaine, pas un toit de chalet. Deux remparts à pic, couverts d'arbres vivaces qui semblent croître sur la tête les uns des autres, nous pressent, nous étreignent, et semblent, par leurs détours multipliés, nous pousser et nous enfermer dans d'inextricables solitudes.

J'ai vu beaucoup de sites plus grandioses, je n'en ai guère vu de plus austères. Les plus belles veines des Alpes, des Pyrénées et des Apennins ne produisent pas une végétation plus robuste et plus imposante ; nulle part je n'ai vu d'aussi belles forêts de sapins gigantesques, élancés, fiers, touffus, et par leur nombre et par leur situation escarpée, semblant braver la destruction et renaître sous les coups de la foudre et de la cognée.

[...] Le torrent se précipite avec fracas derrière la Chartreuse, roule à côté et se laisse tomber sur l'angle d'un bâtiment détaché qu'il achève de dégrader, et qu'il semble prêt à emporter tout à fait dans un jour d'orage. (Lettre X ; p. 894-895; p. 272.)

Le paysage est alors investi d'un sens qui en exprime le sentiment profond, la privation :

Quel était l'emploi de ce bâtiment au temps des moines? Je me suis imaginé que c'était le lieu pénitentiaire, et que la cataracte devait rouler sur la voûte d'un cachot humide et plein de terreur. (Lettre X ; p. 895 ; p. 272.)

Cette association d'idées pittoresques ${ }^{28}$ donne accès au sentiment $\mathrm{du}$ sublime, elle lui est ici absolument nécessaire. Le détour par l'image (ou l'imagerie) convertit la terreur en sublimité. En ce sens, elle met le pittoresque au service du sublime afin de spiritualiser un paysage qui semblait condamné à la déréliction. Le paysage apparaît alors :

Ce site m'a paru ${ }^{29}$, au milieu de la pluie, mélancolique, froid, et admirablement choisi pour une vie éternellement uniforme et pour des hommes voués au culte de l'idée unique et absolue. Point de perspectives, point de contrastes; des pentes de gazon d'un vert égal et magnifique, des profondeurs de forêts sans issue, sans la moindre échappée pour le regard et la pensée ; partout des sapins, des prairies étroites et des forêts coupées par l'invincible rempart de la montagne, par les éternels brouillards... (Lettre X ; p. 895 ; p. 273.)

28. On pourrait également les dire "romanesques ", tant les images sollicitées mêlent dans un même ensemble culturel des références artistiques au sens large du terme (picturales, romanesques, littéraires, etc.) : le roman noir ou gothique, le romantisme frénétique et fantastique, Piranèse, peut-être Sebron ou Granet... Dès le premier tiers du XIX siècle, les deux catégories du "pittoresque " et du "romanesque " vont avoir tendance à se rejoindre pour représenter une seule et même composante du romantisme. Je renvoie sur ce point à l'article de D. Zanone, "Romantiques ou romanesques ? Situer les romans de George Sand ", Littérature, $\mathrm{n}^{\circ} 134$, juin 2004 , p. 5-21, notamment les pages 11-12 dans lesquelles l'examen de Jacques, dont l'écriture est contemporaine des Lettres d'un voyageur, permet de mettre en évidence les liens entre "romanesque ", " pittoresque » et "romantique " dans l'appréhension littéraire et « idéale » du paysage.

29. Sans jeu de mots, on notera combien cette expression est riche de sens. 
Cette apparition du paysage tient autant à la situation objective du site qu'à l'investissement subjectif du contemplateur : le morceau de " pays " devient alors " paysage », c'est-à-dire « situation choisie ou créée par le goût et le sentiment ${ }^{30}$ ". La sensation et le sentiment fondent ainsi ce paysage dans sa sublimité, inscrivent cette apparition fugitive dans l'éternité d'un absolu de la sensation. L'écriture devient ainsi la trace d'un moment, précaire, où le paysage s'enlève dans le sublime. Ce travail complexe d'élaboration descriptive montre combien le paysage sandien se présente comme la « description d'une réalité qui échappe au système de la représentation ${ }^{31} »$. Le Voyageur se plaît alors à souligner avec une certaine ironie romantique combien son travail d'élaboration littéraire a sollicité, par le moyen de l'art pittoresque, les « circonstances accidentelles ${ }^{32}$ » du paysage afin de lui donner un sens et une âme. Le Voyageur présente avec humour et excentricité la fantaisie de son esprit romantique comme une règle académique. Il oppose ainsi au pittoresque de sa description ce qui pourrait bien en être le repoussoir, le poncif:

Je dis éternels, quoique je n'aie passé là qu'une heure. S'ils ne le sont pas, s'il y a jamais un beau soleil sur la Chartreuse de Mériat, si le torrent roule quelquefois limpide et calme, si la tristesse y soulève un instant ses sombres voiles, et si un pareil site s'avise de vouloir sourire, je le déclare poncif, comme on dit dans les ateliers de peinture, c'est-à-dire pleutre, manqué, à côté du beau. Je le déshérite de ma sympathie, je lui retire mon souvenir, et je tiens pour épiciers et malappris tous les voyageurs qui s'y rendront par un beau temps. (Lettre X ; p. 895 ; p. 273.)

Le pittoresque et son pouvoir imageant cadrent l'expression de la fantaisie aussi bien que l'effusion sublime de l'esprit accablé. Les paysages "romantiques " des Lettres d'un voyageur se présentent bien également comme des subversions fantaisistes et des transgressions sublimes de la représentation pittoresque. Chez George Sand, cette synthèse romantique entre le sublime et le pittoresque, l'idéal et le sensible, l'impression subjective et la représentation objective consiste ainsi à faire de l'apparence une apparition.

30. R.-L. de Girardin, De la composition des paysages, op. cit., p. 55 : «Le long des grands chemins, et même dans les tableaux des artistes médiocres, on ne voit que du pays; mais un paysage, une scène poétique, est une situation choisie ou créée par le goût et le sentiment. "

31. P. Laforgue, Romanticoco. Fantaisie, chimère et mélancolie, op. cit., p. 111. Voir aussi, à la même page : «La réalité n'existe pas à l'état de réalité, elle n'existe que dans le mouvement qui en déplie aux yeux du spectateur la représentation. "

32. W. Gilpin, Trois essais sur le beau pittoresque (1792) : «L'œil pittoresque ne se borne pas à examiner la forme et la composition des objets d'un paysage ; il considère aussi les différents effets qu'y produit l'atmosphère, ce vaste et étonnant magasin de la nature. Il n'y a même pas, dans un voyage pittoresque de plus grand plaisir que lorsque l'œil est frappé d'une manière inattendue par une grande scène accompagnée de toutes les circonstances accidentelles de l'atmosphère, qui, lui donnant de l'harmonie, double sa valeur. » Cité dans F. Poussin, "Paysage et représentation. Le relevé d'architecture au XVIII siècle ", Revue des Sciences humaines, $\mathrm{n}^{\circ} 209,1988$, Écrire le paysage, p. 86. 